\title{
Structural Studies of Binary Blend Fibers of Nylon 6 and Nylon 66 by Means of WAXD, SAXS, and DSC
}

\author{
Takehiko Shimomura, Tsunetoshi Matsuda, ${ }^{*}$ and Matsuo Hirami ${ }^{*}{ }^{\dagger}$ \\ Unitika Research Laboratories, Inc., 23 Kozakura, Uji, Kyoto 611-0021, Japan \\ * Research and Development Center, Unitika Ltd., \\ 23 Kozakura, Uji, Kyoto 611-0021, Japan
}

(Received January 28, 1999)

\begin{abstract}
Measurements of wide-angle X-ray diffraction (WAXD), small-angle X-ray scattering (SAXS), and differential scanning calorimetry (DSC) were performed on binary blend fibers of nylon 6 and nylon 66 prepared by drawing and annealing of melt-spun blend fibers. To detect coexisting crystalline phases of both nylon components, we developed a procedure to distinguish $\alpha$-form crystals of nylon 6 and nylon 66 in their mixtures by utilizing higher-order diffraction spots of WAXD for the $\alpha$-form crystals, and applied it to nylon $6 /$ nylon 66 blend fibers over the whole range of composition. Nylons 6 and 66 thus crystallize separately and form individual crystallites of their respective $\alpha$-form crystal structures. By analyzing data of DSC diagram, the appreciable depression of the total degree of crystallinity of both components observed in the intermediate range of composition for unoriented mixture samples reported previously, is almost recovered for drawn and annealed samples treated in this work. The development of crystalline state in the blend fibers brought about by drawing and annealing was confirmed by changes of profiles of SAXS diagram. This tendency is more prominent in nylon 6 rather than nylon 66 . The cause for this is discussed.

KEY WORDS $\alpha$-Form Crystal / Blend Fibers / Crystallization / Differential Scanning Calorimetry / $\gamma$-Form Crystal / Nylon 6/ Nylon 66 / Polymer Blend / Recrystallization / Small-Angle X-Ray Scattering / Wide-Angle X-Ray Diffraction/
\end{abstract}

The features of crystallization and melting in binary mixtures of nylon 6 and nylon 66 have been investigated and the results reported in a study by differential scanning calorimetry (DSC) ${ }^{1}$ in Part I and a study by pressure-volume-temperature $(P V T)$ measurments $^{2}$ in Part II of this series. Although a considerable amount of information on the phase behavior of this mixture system has been obtained, we have been prompted to undertake another study for several reasons. First, in our study on multicomponent systems of crystalline polymers, the scope of the investigation has been confined to binary systems, where two crystallizable polymer components are miscible and crystallize separately, and we assume that this condition is satisfied in nylon $6 /$ nylon 66 mixtures. We wish to confirm the validity of this assumption directly by a structural study.

Secondly, as presented in the previous works for the unoriented system of nylon 6/nylon 66 mixtures, the higher melting temperature component, nylon 66 , crystallizes preferably, while the crystallization of nylon 6 is remarkably restricted, especially in the range where the content of nylon 6 is less than $50 \%$. If the reorganization of morphology becomes possible, by drawing and annealing of unoriented samples of nylon $6 /$ nylon 66 mixtures, it is interesting to examine whether or not nylon 6 molecules frozen in amorphous region will be relaxed and contribute to recrystallization of nylon 6 , and as a result, the different composition dependence of crystalline state will be brought about in drawn and annealed blend fibers compared with that in the unoriented system of mixtures reported previously. ${ }^{1}$

In the present work (Part III of this series), we made measurements of wide-angle X-ray diffraction (WAXD), small-angle X-ray scattering (SAXS), and DSC for

$\dagger$ To whom all correspondence should be addressed. melt-spun, drawn and annealed blend samples of nylon 6 and nylon 66 over the whole range of composition and sought to interpret the results to answer the questions above.

\section{EXPERIMENTAL}

\section{Samples}

As polymer materials in this study, nylon 6 (UnitikaBRL, $\left.M_{w}=16000\right)$ and nylon 66 (BASF-R8270, $M_{w}=$ 22000) were used. Melt-spun monofilament blend samples of nylon 6 and nylon 66 were prepared, where blending ratios of nylon 6 and nylon 66 (N6/N66) are $100 / 0,70 / 30,50 / 50,30 / 70$, and $0 / 100$. The preparation was made by melt-spinning from an extruder at $280^{\circ} \mathrm{C}$ with a take-up speed of $4 \mathrm{mmin}^{-1}$. As confirmed by WAXD pattern, the taken-up monofilament with diameter: $c a .1 .0 \mathrm{~mm}$ is practically unoriented. Subsequently the monofilament sample was drawn by about three times and pressed by a hot-roller at $130^{\circ} \mathrm{C}$ and $150^{\circ} \mathrm{C}$. The sheet-type of sample with thickness: $c a .0 .25 \mathrm{~mm}$ and width: $c a .1 .3 \mathrm{~mm}$ was obtained.

\section{Measurements}

Measurements of WAXD were made by Mac Science model MPX-3 diffractometer with Ni-filtered $\mathrm{Cu}-K_{\alpha}$ radiation of $50 \mathrm{kV}, 32 \mathrm{~mA}$. SAXS were measured by Rigaku Denki model RAD-rB diffractometer with graphite monochromater $\mathrm{Cu}-K_{\alpha}$ radiation of $50 \mathrm{kV}$, $200 \mathrm{~mA}$ by using a small angle goniometer with line collimators at the distance of $300 \mathrm{~mm}$, where dimensions of the first and second slits were $12 \times 0.5$ and $12 \times$ $0.3 \mathrm{~mm}$.

Measurements of DSC were made by Perkin Elmer model DSC-7 in nitrogen atmosphere with scanning speed $10^{\circ} \mathrm{C} \min ^{-1}$. 
( a )
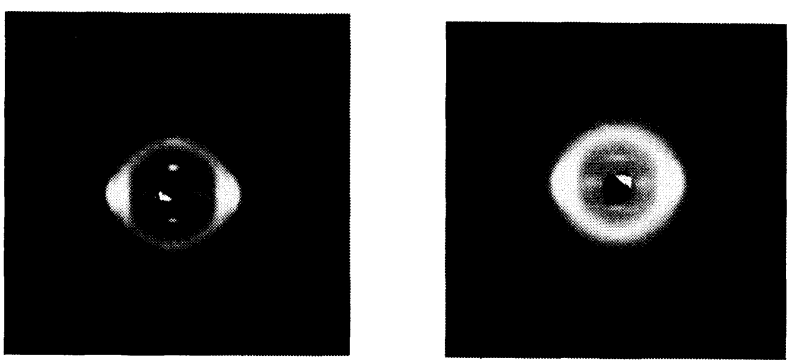

(c)

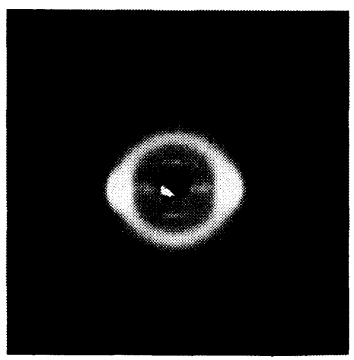

(d)

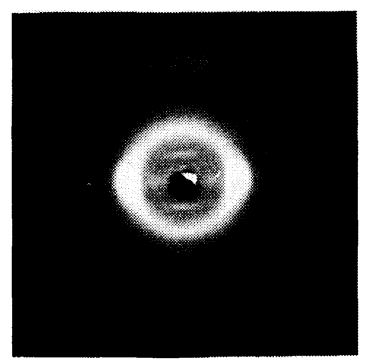

Figure 1. WAXD photographs for binary blend fibers of nylon 6 and nylon 66 (N6/N66). The blending ratio of (N6/N66): (a) 100/0, (b) $70 / 30$, (c) $50 / 50$, (d) $30 / 70$, and (e) $0 / 100$.

\section{RESULTS AND DISCUSSION}

\section{$W A X D$}

In Figure 1, WAXD photographs of drawn and annealed blend fibers of nylon 6 and nylon 66 (N6/N66) are shown, where the blending ratios of $\mathrm{N} 6 / \mathrm{N} 66$ are $100 / 0,70 / 30,50 / 50,30 / 70$, and $0 / 100$. Usually nylon $6^{3}$ and nylon $66^{4}$ crystallize to form stable $\alpha$-form crystals. However, in case of nylon 6 , the $\gamma$-form crystal as polymorphic modification is also formed. ${ }^{5}$ Differences of WAXD pattern between nylon 6 and nylon 66 will be attributed to the conspicuously appeared meridional spot (020) of the $\gamma$-form crystal of nylon 6. In WAXD photographs of the blend fibers as shown in Figure 1, the meridional (020) spot mentioned above can be seen clearly for the sample of N6/N66 $=70 / 30$ and weakly for $\mathrm{N} 6 / \mathrm{N} 66=50 / 50$. The formation of the $\gamma$-form crystal of nylon 6 depends on various crystallization conditions, for example, when quenching from the melt such as ordinary melt spinning of nylon 6 , the $\gamma$-form crystal forms predominantly ${ }^{6}$ and transform to the $\alpha$-form crystal by drawing and/or heat treatment. ${ }^{7}$ From the viewpoint of general applicability, to investigate the coexistence of two crystalline phases of nylon Polym. J., Vol. 31, No. 10, 1999

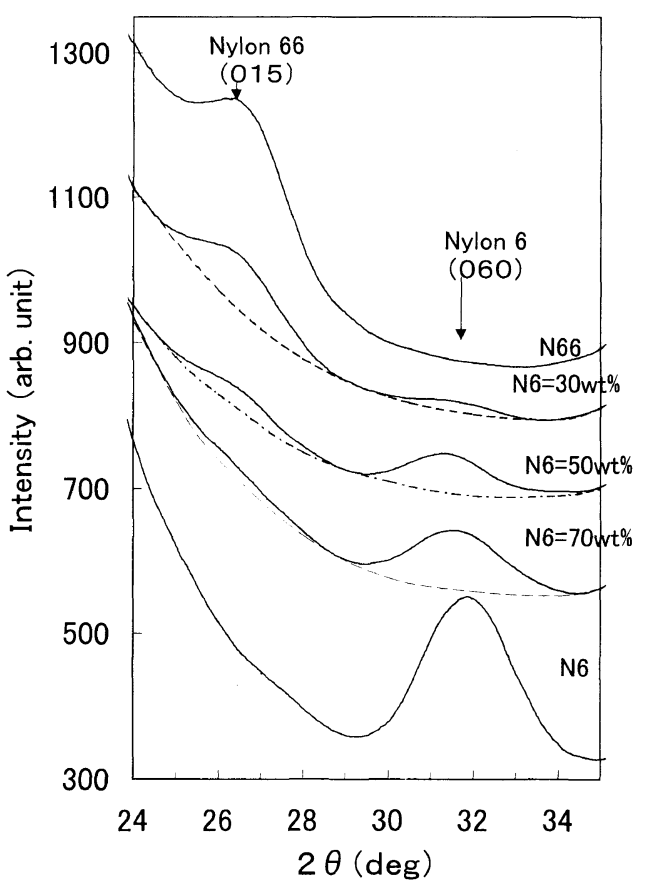

Figure 2. Meridional profiles of WAXD for binary blend fibers (melt-spun, drawn and annealed at $130^{\circ} \mathrm{C}$ ) of nylon 6 and nylon 66 .

6 and nylon 66 in blends, it is desirable to establish a method to distinguish two $\alpha$-form crystal structures of nylon 6 and nylon 66. Since WAXD patterns of the $\alpha$-form crystals are very similar, i.e., their principal reflections are very close, looked for higher-order diffraction spots in each WAXD pattern, appropriate for distinguishing the respective $\alpha$-form crystal structures of nylon 6 and nylon 66 . The (060) spot in the $\alpha$-form crystal of nylon 6 and the (015) spot in the $\alpha$-form crystal of nylon 66 were used. In Figure 2, meridional profiles of WAXD including the two diffraction peaks mentioned above are shown for several blend fiber samples (meltspun, cold-drawn and annealed at $130^{\circ} \mathrm{C}$ ) of nylon 6 and nylon 66. As seen in the figure, the higher-order diffraction spots for the respective $\alpha$-form crystals seem appropriate for our investigation. The peak height of the (060) spot of the $\alpha$-form crystal of nylon 6 decreases as the content of nylon 6 decreases, but even at $\mathrm{N} 6=30 \%$, the profile of the $(060)$ spot is recognized appreciably.

It is to be emphasized that the result obtained shown in Figure 2 strongly supports the validity of a basic assumption of the present work, that is, nylon 6 and nylon 66 in mixtures crystallize separately and form individual crystallites of the respective crystal structures. Thus, the possibility of cocrystallization is almost excluded.

\section{DSC}

In Figure 3, DSC diagrams for drawn and annealed blend fibers of N6/N66 are shown where blending ratios of N6/N66 are 100/0,70/30,50/50,30/70, and 0/100. As for the melting profile of nylon 66 , the peak height and melting temperature read at the peak position decrease, as the content of nylon 6 increases. The melting temperature depression of nylon 66 observed here is smaller than that for the unoriented system of binary 


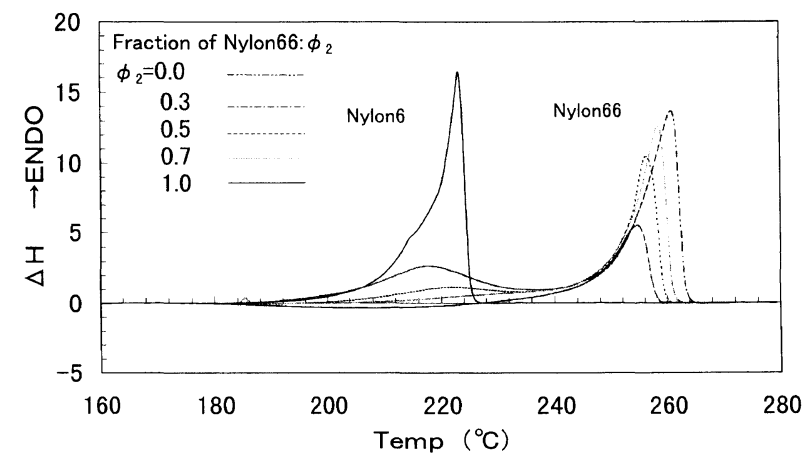

Figure 3. DSC diagram for binary blend fibers (melt-spun, drawn and annealed at $130^{\circ} \mathrm{C}$ ) of nylon 6 and nylon 66 .

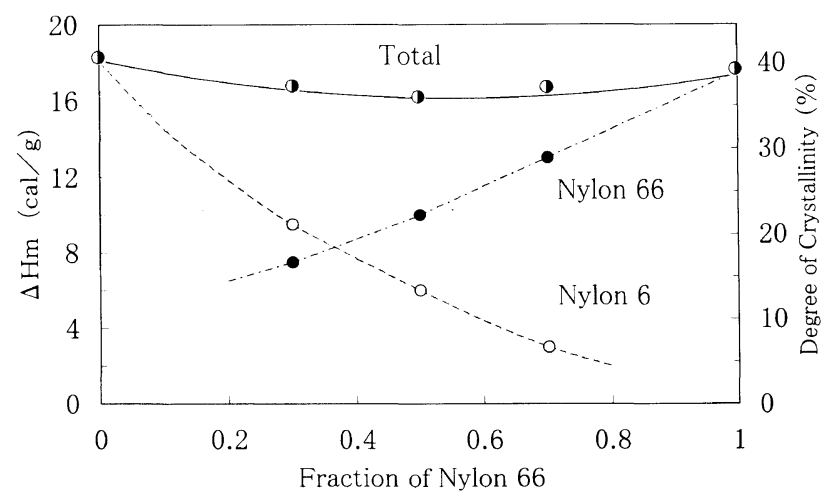

Figure 4. Plots of observed $\Delta H_{\mathrm{m}}$ against composition for binary blend fibers (melt-spun, drawn and annealed at $130^{\circ} \mathrm{C}$ ) of nylon 6 and nylon 66.

mixtures of nylon 6 and nylon 66 reported previously. ${ }^{1}$ The reason for this difference of melting temperature depression is due to differences of degree of mixing of two components between the two series of mixture samples. In the present work, the preparation of mixture samples were made by blending two different nylon pellets in an extruder and melt-spun blend fibers were submitted to subsequent procedures of cold-drawing and annealing. The degree of mixing in this series of blend fiber samples thus obtained is insufficient for the molecularly mixing state.

The heat of fusion $\Delta H_{\mathrm{m}}$ for each of nylon 6 and nylon 66 in blend fibers is obtained from the area under the melting profile in DSC diagram of Figure 3. Since the heats of fusion of nylon 6 and nylon 66 are the same, i.e., $45 \mathrm{cal} \mathrm{g}^{-1}, 8$ the degree of crystallinity $\%$ can be calculated from the observed value of $\Delta H_{\mathrm{m}}\left(\mathrm{cal} \mathrm{g}^{-1}\right)$ by the relation of $\left(\Delta H_{\mathrm{m}} / 45\right) \times 100 . \Delta H_{\mathrm{m}}$ of (i) nylon 6 , (ii) nylon 66 and (iii) their sum are shown as functions of composition in Figure 4, in which the three cases of (i), (ii), and (iii) are indicated by the symbols; $\bigcirc, \bigcirc$, and () respectively. Note that the degree of crystallinity is shown by the right vertical axis in Figure 4 . In the previous paper, ${ }^{1}$ in DSC diagram for the unoriented system of binary mixtures of nylon 6 and nylon 66, the total degree of crystallinity decreases markedly in the intermediate region of composition, by $25-30 \%$ relatively compared to the pure nylon 66 or nylon 6 . As seen in Figure 4, such depression of the total crystallinity in the intermediate region of composition reported previously ${ }^{1}$ is almost recovered in the present system of

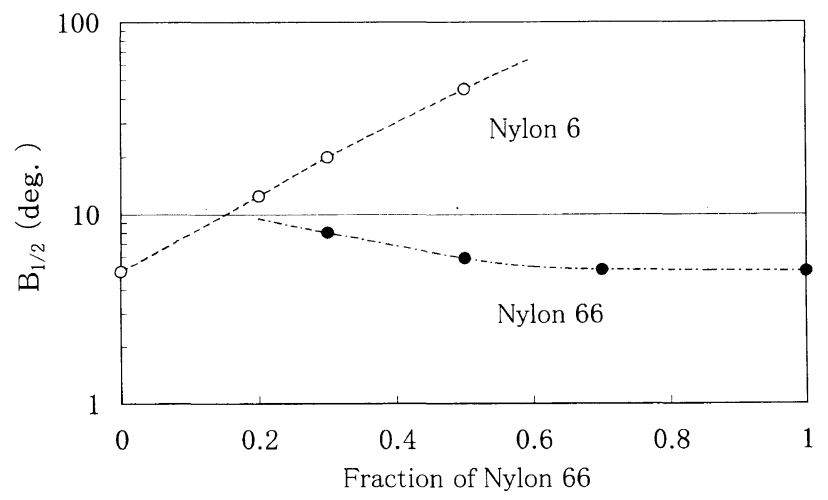

Figure 5. Plots of parameter $B_{1 / 2}$ for nylon 6 and nylon 66 against composition.

binary blend fibers of nylon 6 and nylon 66 .

As for the crystallization of binary mixtures of nylon 6 and nylon 66 in unoriented state, on the cooling process from the melt, nylon 66, the higher melting temperature component, crystallize preferably, and nylon 6 crystallizes only in the intervening space surrounded by pre-crystallized region of nylon 66. The crystallization of nylon 6 is remarkably restricted, especially in the range where the content of nylon 6 is less than $50 \%$. However, when unoriented mixture samples are submitted to drawing and the subsequent annealing, morphology of nylon 6/nylon 66 mixtures as mentioned above is reorganized significantly. In this case, nylon 6 molecules frozen in the amorphous region become relaxed, and join in the re-crystallization process of nylon 6 . In terms of this reorganized morphology, we understand the behavior that the inferiority of crystallinity of nylon 6 has been improved markedly.

Concerning the broadness of melting temperature profile in DSC diagram, we introduced a parameter $B_{1 / 2}$ defined as the half-width of a peak for melting temperature profile, and the plot of $B_{1 / 2}$ against composition is shown in Figure 5. As seen, in case of nylon $6, B_{1 / 2}$ increases remakably, while $B_{1 / 2}$ of nylon 66 stays and increases slightly after the content of nylon 66 becomes less than $50 \%$. Although not exactly defined as a parameter on the quantitative basis, $B_{1 / 2}$ is useful for comparative purposes, since it may represent relatively the broadness of crystal thickness distribution and also the disorderness of crystalline state. It is to be noted that the shape of melting temperature profile is not symmetrical but has a long skew tail in lower temperature side. Thus, the contribution to the overlapping portion of two melting temperature profiles is mainly from the lower temperature side-tail of nylon 66 rather than the high temperature side-tail of nylon 6. Based on the consideration as described above, broad melting temperature profiles of nylon 6 in the intermediate region of mixtures suggest that crystallites formed during the course of recrystallization may have a broad distribution of thickness including small size crystals. This is not surprising, since the structure formation of nylon 6 molecules during recrystallization proceeds under very restricted circumstances.

\section{$S A X S$}

SAXS meridional profiles of melt-spun, cold-drawn 
(a)

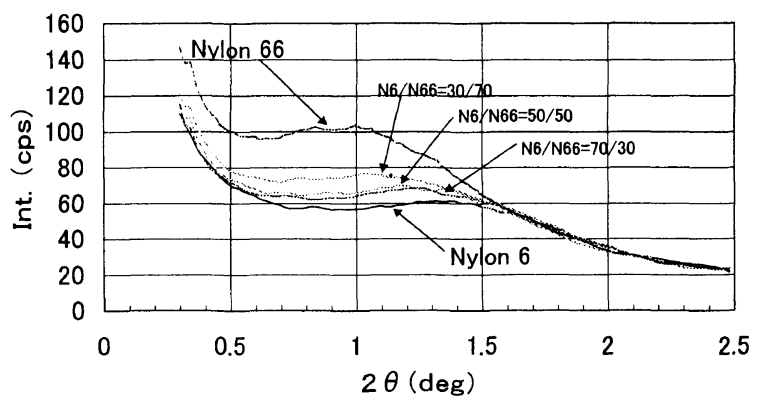

(b)

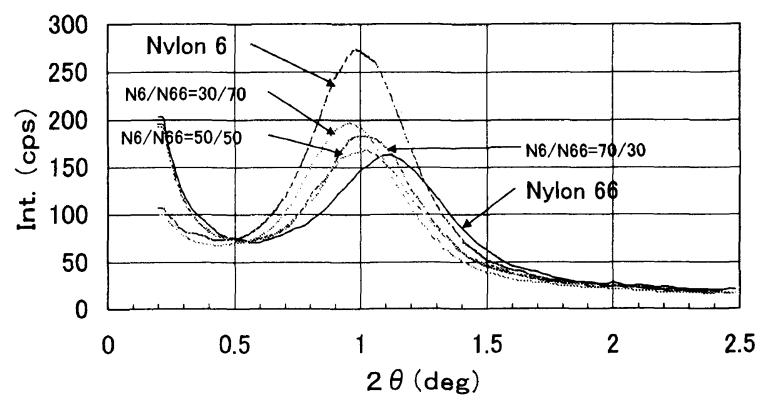

(c)

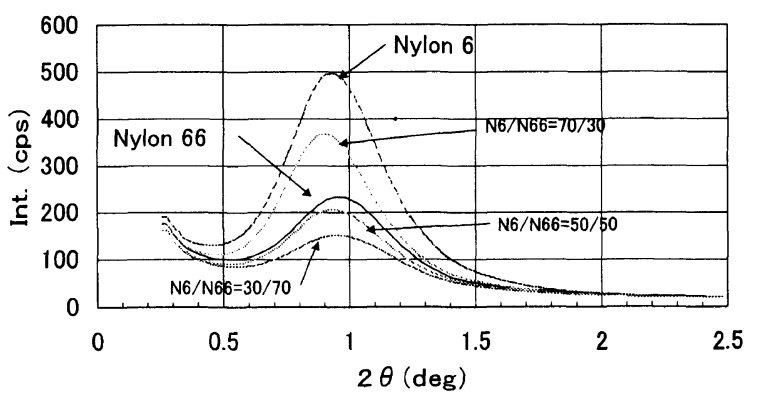

Figure 6. SAXS patterns for binary blend fibers of nylon 6 and nylon 66. (a) undrawn fibers, (b) drawn fibers, annealed at $130^{\circ} \mathrm{C}$, and (c) drawn fibers, annealed at $150^{\circ} \mathrm{C}$.

(by three times) and annealed (at $130^{\circ} \mathrm{C}$ and $150^{\circ} \mathrm{C}$ ) blend fibers are shown in Figures 6(a), 6(b), and 6(c); namely, (a) undrawn fibers (control), (b) annealed at $130^{\circ} \mathrm{C}$, (c) annealed at $150^{\circ} \mathrm{C}$.

Changes of SAXS pattern from Figure 6(a) to Figures 6(b), and 6(c), i.e., shapes of profiles become sharper and the SAXS-spacing read at the peak position increases, represents the reorganization of morphology in. blend fibers of nylon 6 and nylon 66 during drawing and annealing and the extent of change is more prominent in nylon 6 than nylon 66. In Figure 6(a), nylon 66 exhibits maximum peak height, while nylon 6 the minimum. In Figures 6(b) and 6(c), nylon 6 exhibits the maximum peak height. From SAXS pattern in Figure 6(a) to that in Figure 6(c), the SAXS-spacing of nylon 66 increases from $80 \AA$ to $90 \AA$, a relative increase by $10 \%$, while that of nylon 6 increases from $65 \AA$ to $95 \AA$, a relative increase by $45 \%$.

The results above can be interpreted systematically based on the behavior of crystallization and recrystalliza- tion of nylon 6 and nylon 66 in blends, discussed in detail in the previous section. During drawing and annealing of the undrawn fibers, the contribution of recrystallization of nylon 6 molecules is significant.

It is to be noted that as for the relation between the rate of crystal growth of nylon 6 and crystallization temperature, according to literature data reported early by Burnett and McDevit, ${ }^{9}$ and later by Magil, ${ }^{10}$ the maximum rate lies around the crystallization temperature of $140^{\circ} \mathrm{C}$. This is related to the behavior of recrystallization of nylon 6 , that is, the remarkable enhancement of crystalline state seen in Figures 6(b) and 6(c).

In conclusion, the features of SAXS pattern for drawn and annealed blend fibers of nylon 6 and nylon 66 compared with the corresponding undrawn fibers are quite similar with those of DSC diagram for the same series of samples presented in the previous section. Experimental data could be understood from the crystallization and recrystallization of nylon 6 and nylon 66 in blend fibers.

\section{CONCLUSION}

Measurements of WAXD, DSC, and SAXS on binary blend fibers (melt-spun, cold-drawn and annealed) of nylon 6 and nylon 66 were performed and the following conclusions were obtained.

(1) To detect coexisting crystalline phases of nylon 6 and nylon 66 in mixtures, we developed a procedure to distinguish $\alpha$-form structures of both nylons by higher-order diffraction spots of WAXD. The procedure was applied to several nylon 6/nylon 66 blend fibers and two higher-order diffraction spots (employed as index for both nylons) in meridional profile of WAXD were clearly observed separately. This supports the validity of the assumption that nylon 6 and nylon 66 crystallize separately in their mixtures.

(2) From melting profiles in DSC diagram, the degree of crystallinity of each component and total crystallinity were evaluated as functions of composition. The depression of total crystallinity (by $25-30 \%$ compared to the pure nylon 6 or nylon 66) observed in the intermediate range of composition for the unoriented mixture samples reported previously, almost recovers for the oriented blend fibers treated here. The reason is attributed mainly to the contribution of recrystallization of nylon 6 during reorganization of morphology by drawing and annealing. Broad melting profiles in DSC diagram for nylon 6 in the mixtures suggest that nylon 6 crystals formed by recrystallization have a broad distribution of thickness including small size crystals.

(3) The development of crystalline state toward the higher level brought about by drawing and annealing in the blend fibers was confirmed by changes of profiles of SAXS diagram, namely the shapes of profiles become sharper, peak height increases and the SAXS-spacing increases. This is more prominent in nylon 6 rather than nylon 66 , and is caused by the crystallization and recrystallization of nylon 6 and nylon 66. Especially, the recrystallization of nylon 6 plays a significant role.

Acknowledgment. This work was supported by the 
international joint research grant of NEDO (New Energy and Industrial Technology Development Organization). The authors are grateful to Prof. K. Kaji and Dr. M. Tsuji for helpful discussion and comments.

\section{REFERENCES}

1. T. Matsuda, T. Shimomura, and M. Hirami, Polym. J., 31, 795 (1999).

2. K. Matsumura, T. Shimomura, T. Matsuda, and M. Hirami, Polym. J., 31, 836 (1999).

3. C. W. Bunn and E. V. Garnar, Proc. R. Soc. Lond. A, 189, 39 (1947)
4. D. R. Holmes, C. W. Bunn, and D. J. Smith, J. Polym. Sci., 17, 159 (1955).

5. H. Arimoto, M. Ishibashi, M. Hirai, and Y. Chatani, J. Polym. Sci. A, 3, 317 (1965).

6. J. L. White and M. Cakmak, Adv. Polym. Tech., 6, 295 (1986), p 328.

7. K. Miyasaka and K. Ishikawa, J. Polym. Sci., A2, 6, 1317 (1968).

8. L. Mandelkern, "Crystallization of Polymers," McGraw-Hill, New York, N.Y., 1963, Chapter 5-2, p 120.

9. B. B. Burnett and W. I. F. McDevit, J. Appl. Phys., 28, 1101 (1957).

10. J. H. Magill, Polymers, 6, 367 (1965). 